\title{
Exercício físico e hábitos alimentares em idosos: uma reflexão teórica
}

\author{
Physical exercise and eating habits in the elderly: a theoretical reflection \\ Ejercicio físico y hábitos alimentarios en el anciano: una reflexión teórica
}

Recebido: 07/12/2021 | Revisado: 14/12/2021 | Aceito: 14/12/2021 | Publicado: 21/12/2021

\author{
Raul José de Araújo Medeiros \\ ORCID: https://orcid.org/0000-0003-3404-8136 \\ Faculdade Independente do Nordeste, Brasil \\ E-mail: RaulCezar1996@hotmail.com \\ Luciana Araújo dos Reis \\ ORCID: https://orcid.org/0000-0002-0867-8057 \\ Universidade Estadual do Sudoeste da Bahia, Brasil \\ E-mail: lucianauesb@yahoo.com.br \\ Flávia Rocha Brito \\ ORCID: https://orcid.org/ 0000-0002-7273-4957 \\ Faculdade Pitágoras de Medicina, Brasil \\ E-mail: flaviarrbrito@gmail.com \\ Wagner Couto Assis \\ ORCID: https://orcid.org/0000-0001-7802-2443 \\ Universidade Estadual do Sudoeste da Bahia, Brasil \\ E-mail: wagnerassis2010@ hotmail.com \\ Frank Evilácio de Oliveira Guimarães \\ ORCID: https://orcid.org/0000-0003-4739-3676 \\ Universidade Católica do Salvador, Brasil \\ E-mail: frankevilacio@ hotmail.com \\ Sílvio Andrade Melo \\ ORCID: https://orcid.org/0000-0002-2386-9724 \\ Faculdade de Tecnologia e Ciências, Brasil \\ E-mail: silvio_amelo@hotmail.com \\ Luana Araújo dos Reis \\ ORCID: https://orcid.org/0000-0002-9263-083X \\ Faculdade Independente do Nordeste, Brasil \\ Instituto Multidisciplinar em Saúde, Brasil \\ E-mail: luareis1@ hotmail.com
}

\begin{abstract}
Resumo
Objetivo: Analisar, a partir da literatura, a prática de exercício físico e hábitos alimentares nos Idosos. Metodologia: Trata-se de um ensaio teórico de caráter reflexivo, realizado por meio de uma revisão bibliográfica não sistemática, com abordagem qualitativa. A coleta dos dados foi realizada através de busca online dos artigos nas bases de dados: google acadêmico, biblioteca eletrônica científica online (SCIELO), centro nacional de informações sobre biotecnologia (NCBI) e PUBMED, utilizando os descritores: exercícios físicos, hábitos alimentares, idosos, bemestar, envelhecimento, e suas respectivas traduções padronizadas nos descritores em ciências da saúde (DECS). Como critérios de inclusão para seleção dos materiais foram considerados: artigos nos idiomas inglês e português, publicados no período de 2010 a 2020, disponíveis na integra e cujos resultados abrangessem o tema desta pesquisa. Resultados: A análise da literatura foi disposta em cinco categorias temáticas, a saber: Processo de envelhecimento; Envelhecimento saudável; Exercício físico; Qualidade de vida da pessoa idosa; e, Hábitos alimentares. Considerações Finais: A prática de exercício físico e hábitos alimentares saudáveis devem ser estimuladas nos Idosos, tendo em vista seu comprovado impacto na qualidade de vida destes.
\end{abstract}

Palavras-chave: Exercícios físicos; Hábitos alimentares; Idosos; Bem-estar; Envelhecimento.

\section{Abstract}

Objective: To analyze, based on the literature, the practice of physical exercise and eating habits in the elderly. Methodology: This is a theoretical essay of a reflective nature, carried out through a non-systematic bibliographic review, with a qualitative approach. Data collection was performed through an online search of articles in databases: academic google, online scientific electronic library (SCIELO), national center for information on biotechnology (NCBI) and PUBMED, using the descriptors: physical exercises, eating habits, elderly, well-being, aging, and their respective standardized translations in health sciences descriptors (DECS). As inclusion criteria for the selection of materials, the following were considered: articles in English and Portuguese, published from 2010 to 2020, available in full and whose results covered the topic of this research. Results: The literature analysis was arranged in five thematic categories, namely: Aging process; Healthy aging; Physical exercise; Quality of life of the elderly person; 
and, Eating habits. Final Considerations: The practice of physical exercise and healthy eating habits should be encouraged in the Elderly, in view of its proven impact on their quality of life.

Keywords: Physical exercises; Eating habits; Seniors; Welfare; Aging.

\section{Resumen}

Objetivo: Analizar, a partir de la literatura, la práctica de ejercicio físico y hábitos alimentarios en ancianos. Metodología: Se trata de un ensayo teórico de carácter reflexivo, realizado a través de una revisión bibliográfica no sistemática, con un enfoque cualitativo. La recolección de datos se realizó mediante búsqueda en línea de artículos en bases de datos: google académico, biblioteca electrónica científica en línea (SCIELO), centro nacional de información en biotecnología (NCBI) y PUBMED, utilizando los descriptores: ejercicios físicos, hábitos alimenticios, ancianos, bienestar, envejecimiento, y sus respectivas traducciones estandarizadas en descriptores de ciencias de la salud (DECS). Como criterios de inclusión para la selección de materiales, se consideraron los siguientes: artículos en inglés y portugués, publicados de 2010 a 2020, disponibles en su totalidad y cuyos resultados cubrieron el tema de esta investigación. Resultados: El análisis de la literatura se organizó en cinco categorías temáticas, a saber: proceso de envejecimiento; Envejecimiento saludable; Ejercicio físico; Calidad de vida del anciano; y Hábitos alimenticios. Consideraciones finales: Se debe fomentar la práctica de ejercicio físico y hábitos alimenticios saludables en las personas mayores, dado el impacto comprobado en su calidad de vida.

Palabras clave: Ejercicios físicos; Hábitos alimenticios; Personas mayores; Bienestar; Envejecimiento.

\section{Introdução}

O processo de envelhecimento é um percurso progressivo que ocorre durante a vida afetando todos os organismos, resultando em alterações dos padrões fisiológicos de um indivíduo, em uma relação mútua de fatores sociais, culturais, biológicos e psicológicos. O conceito de envelhecer pode ser entendido como algo subjetivo e de transformações biopsicossociais que modificam aspectos comuns em indivíduos saudáveis, levando-os a novas percepções de enfrentamento da vida (Cancela, 2017).

Mesmo correspondendo a um processo natural do ser humano, nem todas as pessoas gozam do envelhecimento saudável, países desenvolvidos apresentam melhores indicadores comparados aos em desenvolvimento, contudo o interesse por ter vidas mais longas, inerentemente relacionado à busca pela saúde, é uma realidade da sociedade atual (OPAS, 2020).

A Organização Mundial de Saúde (OMS, 2015) menciona a amplitude de oportunidades que vem ajudando no aumento da longevidade, porém os seus impactos dependerão da saúde do idoso. Se as pessoas vivenciam a velhice se cuidando, cuidando da sua saúde e sendo produtivos, serão idosos fortes e independentes.

Cabe mencionar que, neste contexto, a alimentação exerce grande influência sobre cada pessoa, principalmente sobre a sua saúde, sua capacidade de trabalhar, estudar, divertir-se, sua aparência e sua longevidade. A nutrição é um dos fatores comportamentais mais importantes que afetam o estado de saúde de um indivíduo ou de uma nação (Salgado, 2004).

Para além da alimentação adequada, o hábito da prática de atividade física e educativa proporciona aos idosos a serem mais ativos, melhora a saúde, motiva para a autonomia e a valorizarem mais as suas capacidades (Flores et al., 2018). Praticar exercícios físicos regularmente permite ao idoso sentir-se mais disposto, aumenta à vontade para realizar tarefas e apresenta mais vontade de viver (Azevedo Filho, 2018).

Estudo realizado por Prado et al. (2021) refere que a prática de exercício físico para a saúde do idoso traz muitos benefícios, dentre eles cita-se a melhora nas capacidades cognitivas e funcionais, redução no risco de doenças crônicas e incapacitantes, aumento da qualidade de vida e bem-estar, além de ser responsável pela inserção do idoso nos ambientes sociais.

Neste contexto, esta pesquisa apresenta como objetivo analisar, a partir da literatura, a prática de exercício físico e hábitos alimentares nos Idosos. 


\section{Metodologia}

Este é um ensaio teórico de caráter reflexivo, realizado por meio de uma revisão bibliográfica não sistemática, com abordagem qualitativa. Gil (2017) refere que a pesquisa bibliográfica permite analisar uma temática sob diversos estudos de maneira ampla e concisa. Já a abordagem qualitativa responde a questões muito particulares. Ela se preocupa, nas ciências sociais, com um nível de realidade que não pode ser quantificado. Ou seja, ela trabalha com o universo de significados, motivos, aspirações, crenças, valores e atitudes, o que corresponde a um espaço mais profundo das relações, dos processos e dos fenômenos que não podem ser reduzidos à operacionalização de variáveis (Minayo, 2001 apud Ferraz et al., 2021, p. 3).

A coleta dos dados foi realizada através de busca online dos artigos nas bases de dados: google acadêmico, biblioteca eletrônica científica online (SCIELO), centro nacional de informações sobre biotecnologia (NCBI) e PUBMED, utilizando os descritores: "exercícios físicos", "hábitos alimentares", "idosos", "bem-estar", "envelhecimento", e suas respectivas traduções padronizadas nos descritores em ciências da saúde (DECS). Como critérios de inclusão para seleção dos materiais foram considerados: artigos nos idiomas inglês e português, publicados no período de 2010 a 2020, disponíveis na integra e cujos resultados abrangessem o tema desta pesquisa.

Os resultados foram organizados em cinco categorias temáticas, a saber: Processo de envelhecimento; Envelhecimento saudável; Exercício físico; Qualidade de vida da pessoa idosa; e, Hábitos alimentares.

Foram atendidos os aspectos éticos de acordo com a Lei $\mathrm{n}^{\circ}$ 9.610, de 19 de fevereiro de 1998, que dispõe sobre direitos autorais. Por se tratar de pesquisa de revisão de literatura não será necessária a submissão ao comitê de ética.

\section{Resultados e Discussão}

\section{Processo de Envelhecimento}

O Programa de Atenção Integrada para a Pessoa Idosa (ICOPE) foi desenvolvido pela Organização Mundial da Saúde (OMS) para atender às necessidades e demandas de saúde das populações idosas em todo o mundo. Em 2050, a proporção da população global com 60 anos ou mais quase dobrará. Nas Américas, a expectativa de vida aumentou 21,6 anos nas últimas seis décadas. No entanto, viver mais frequentemente significa enfrentar problemas de saúde acumulados durante a velhice (OPAS, 2020).

O envelhecimento pode variar de indivíduo para indivíduo, sendo gradativo para uns e mais rápido para outros. Essas variações são dependentes de fatores como estilo de vida, condições socioeconômicas e doenças crônicas. Já o conceito "biológico" relaciona-se com aspectos nos planos molecular, celular, tecidual e orgânico do indivíduo, enquanto o conceito psíquico é a relação das dimensões cognitivas e psicoafetivas, interferindo na personalidade e afeto. Desta maneira falar de envelhecimento é abri o leque de interpretações que se entrelaçam ao cotidiano e a perspectivas culturais diferentes (Caetano, 2006).

O envelhecimento é um fenômeno que atinge todos os seres humanos, independentemente. Sendo caracterizado como um processo dinâmico, progressivo e irreversível, ligados intimamente a fatores biológicos, psíquicos e sociais (Brito \& Litvoc, 2004).

Fala-se correntemente do envelhecimento como se tratando de um estado tendencialmente classificado de "terceira idade" ou ainda "quarta idade". No entanto, o envelhecimento não é um estado, mas sim um processo de degradação progressiva e diferencial. Ele afeta todos os seres vivos e o seu termo natural é a morte do organismo. É, assim, impossível datar o seu começo, porque de acordo com o nível no qual ele se situa (biológico, psicológico ou sociológico), a sua velocidade e gravidade variam de indivíduo para indivíduo. Assim, podemos dizer que os indivíduos envelhecem de formas muito diversas e, a este respeito, podemos falar de idade biológica, de idade social e de idade psicológica, que podem ser muito diferentes da idade cronológica (Fontaine, 2000). 
Moraes e Lima (2010) trazem em seu estudo a divisão do envelhecimento em dois aspectos: biológico e psíquico. O envelhecimento biológico é irreversível, causando vulnerabilidade do organismo, e é de natureza multifatorial, dependendo da programação genética e alterações que ocorrem no nível celular. Já o envelhecimento psíquico ou amadurecimento depende do esforço pessoal na busca do autoconhecimento; a pessoa idosa torna-se capaz de tolerar a dor ou a perda da independência biológica, e assim ele não é naturalmente progressivo.

O envelhecimento fisiológico compreende uma série de alterações nas funções orgânicas e mentais devido exclusivamente aos efeitos da idade avançada sobre o organismo, fazendo com que o mesmo perca capacidade de manter o equilíbrio homeostático e que todas as funções fisiológicas gradualmente comecem a declinar. Tais alterações têm por características principal a diminuição progressiva da reserva funcional. Ou seja, um organismo envelhecido, em condições normais, poderá sobreviver adequadamente, porém, quando submetido a situações de stress físico, emocional, etc., pode apresentar dificuldades em manter sua homeostase e, desta forma, manifestar sobrecarga funciona, a qual pode culminar em processos patológicos, uma vez que há o comprometimento dos sistemas endócrino, nervoso e imunológico (Firmimo, 2006).

O envelhecimento perceptivo é muito diferenciado. Algumas modalidades sensoriais, como o olfato, o gosto ou a cinestesia, são pouco afetadas pela idade, ao passo que outras, como a audição, a visão e o equilíbrio, são gravemente afetadas. De todas estas modalidades perceptivas, o envelhecimento afeta de forma mais significativa o equilíbrio, audição e visão, sendo que isto acarreta consequências importantes, e por vezes graves, a nível psicológico e social. Por outro lado, os défices sensoriais de natureza auditiva e visual parecem causas importantes de declínio geral no funcionamento das atividades intelectuais (Fontaine, 2000).

Um dos principais fatores que contribui para um envelhecimento saudável está associado diretamente ao conjunto de relações pessoais e influências ambientais em que o idoso está inserido, ou seja, indivíduos que passam exatamente pelas mesmas condições, sejam elas negativas ou positivas, podem apresentar posturas e comportamentos completamente diferentes (Santos et al., 2016).

\section{Envelhecimento Saudável}

Até 2025, segundo a OMS, o Brasil será o sexto país do mundo em número de idosos. Ainda é grande a desinformação sobre a saúde do idoso e as particularidades e desafios do envelhecimento populacional para a saúde pública em nosso contexto social. Entre 1980 e 2000 a população com 60 anos ou mais cresceu 7,3 milhões, totalizando mais de 14,5 milhões em 2000. O aumento da expectativa média de vida também aumentou acentuadamente no país. Este aumento do número de anos de vida, no entanto, precisa ser acompanhado pela melhoria ou manutenção da saúde e qualidade de vida. O Programa do Ministério da Saúde "Brasil Saudável” envolve uma ação nacional para criar políticas públicas que promovam modos de viver mais saudáveis em todas as etapas da vida, favorecendo a prática de atividades físicas no cotidiano e no lazer, o acesso a alimentos saudáveis e a redução do consumo de tabaco. Estas questões são a base para o envelhecimento saudável, um envelhecimento que signifique também um ganho substancial em qualidade de vida e saúde (WHO, 2005).

Ainda segundo a Organização Mundial de Saúde, o envelhecimento ativo é o processo de otimização das oportunidades de saúde, participação e segurança, com o objetivo de melhorar a qualidade de vida à medida que as pessoas ficam mais velhas. $\mathrm{O}$ envelhecimento ativo aplica-se tanto a indivíduos quanto a grupos populacionais. Permite que as pessoas percebam o seu potencial para o bem-estar físico, social e mental ao longo do curso da vida, e que essas pessoas participem da sociedade de acordo com suas necessidades, desejos e capacidades; ao mesmo tempo, propicia proteção, segurança e cuidados adequados, quando necessários.

A palavra "ativo" refere-se à participação contínua nas questões sociais, econômicas, culturais, espirituais e civis, e não somente à capacidade de estar fisicamente ativo ou de fazer parte da força de trabalho. O objetivo do envelhecimento ativo 
é aumentar a expectativa de uma vida saudável e a qualidade de vida para todas as pessoas que estão envelhecendo, inclusive as que são frágeis, fisicamente incapacitadas e que requerem cuidados (WHO, 2005).

O termo "envelhecimento ativo" foi adotado pela Organização Mundial da Saúde no final dos anos 90. Procura transmitir uma mensagem mais abrangente do que "envelhecimento saudável", abrangente do que "envelhecimento saudável", e reconhecer, além dos cuidados com a saúde, outros fatores que afetam o modo como os indivíduos e as populações envelhecem (Kalache \& Kickbush, 1997).

O paradigma do envelhecimento ativo traz consigo um novo enfoque, assente na construção de um mundo muito mais favorável aos adultos mais velhos, solicitando uma intervenção mais ampla do Estado que se quer de bem-estar. E, nesse quadro, as políticas sociais e culturais são eixos estruturantes das intervenções públicas dirigidas às populações. Tendo nós consciência de que as políticas públicas são reflexos da forma como o sistema de proteção social percepciona os direitos sociais dos cidadãos no geral, e dos diversos grupos em particular, reiteramos que, neste cenário pós-moderno, "as políticas devem ser estruturadas de forma que permitam um maior número de pessoas alcançarem trajetórias positivas do envelhecimento" (OMS, 2015, p. 6).

De acordo com a Estratégia Nacional para o Envelhecimento Ativo e Saudável (Brasil, 2017), é muito importante que os adultos mais velhos continuem a sentir-se parte das diversas instituições comunitárias, designadamente as culturais. Nesse sentido, deve-se apostar na criação de ambientes potenciadores da integração e da participação, pois a participação em atividades de lazer, sociais, culturais e espirituais realizadas no âmbito da comunidade e da família permite aos idosos continuar a exercer as suas competências, a ser objeto de respeito e estima e a manter ou estabelecer relações de apoio e de afeto (OMS, 2009).

\section{Exercício Físico}

O exercício físico é uma atividade planejada. São movimentos executados de maneira planejada e que possuem um objetivo específico. Precisando de constância e um profissional especifico para orientar a pratica da atividade. Por mais que o princípio da individualidade afete o nível de perda funcional, estudos apontam a média de diminuição da força muscular de 20 a 40\% em idosos entre 70 e 80 anos. Aos 90 anos, esta taxa pode ser maior que 50\% (Garcia, 2008; Perfeito, 2014). Esta degradação da força atrelada a perda de massa muscular tem sido relatada como preditora para o aumento da dependência funcional, interferindo diretamente na qualidade de vida do indivíduo (Doherty et al., 2003; Silva et al., 2006; Hanson et al., 2009; Ara, 2015).

Shephard (1990) enumera melhora da saúde, aumento da oportunidade de contatos sociais e ganhos na função cerebral como as principais razões para que indivíduos sedentários há anos, repentinamente, aos 70 ou 80 anos de idade, iniciem e mantenham a prática de exercícios físicos. As vantagens da prática de exercícios para idosos dependem de como se processa o envelhecimento e da rotina de exercício físico praticada.

E $1^{\circ}$ de outubro de 2003 foi instituída a Lei 10.741, que criou o Estatuto do Idoso, uma referência para as políticas públicas sociais e de saúde, sendo este o elemento primordial para a qualidade de vida. Em outubro de 2006 foi assinada a portaria $n^{\circ} 2.528$ do Ministério da Saúde que implementou a Política Nacional de Saúde do Idoso (PNSPI), que tem por objetivo garantir um envelhecimento saudável, que significa preservar a capacidade funcional, autonomia e manter o nível de qualidade de vida. Estes instrumentos são considerados legais e indispensáveis à inclusão funcional de idosos e fazem parte das diretrizes à promoção do envelhecimento ativo e saudável (Brasil, 2006).

A terceira idade, para muitos, tem início a partir dos 60 anos de idade. É uma expressão que, segundo Silva, popularizou-se com muita rapidez no vocabulário, onde o seu uso não é explicado pela referência a uma idade cronológica precisa, mas por ser essa uma forma de tratamento das pessoas de mais idade, que não adquiriu ainda uma conotação 
depreciativa, como aconteceu com o termo idoso, que ainda é muito vinculada à imagem do tipo de idoso incapaz, dependente, muito velho e doente, prostrado em uma cadeira, esquecido do mundo (Laslett, 1987).

Na concepção de Ferretti (1987), pode-se considerar a velhice sob três aspectos: o cronológico quando a pessoa atinge 65 anos, o funcional referente às limitações e incapacidades, o vital, considerado um conceito mais moderno, coloca que, nesta etapa da vida, as potencialidades são únicas e distintas, deve-se levar em conta a serenidade, a experiência, a maturidade, a perspectiva de vida pessoal e social.

\section{Hábitos Alimentares}

Uma alimentação adequada exerce um papel importantíssimo para a prevenção, manutenção e recuperação da saúde do idoso, atenuando as alterações sofridas. Essa deve ser equilibrada, pobre em gorduras saturadas e trans, frituras, e rica em alimentos naturais, vegetais, frutas e legumes, fibras, vitaminas e minerais (Sanavita, 2003).

A alimentação adequada, para os idosos, deve constar de uma alimentação equilibrada com proteínas, vitaminas, e sais minerais; com baixo teor de gordura; rica em fibras; moderada em sal e açúcar; conter líquidos; e serem efetuadas em menor quantidade, mas em maior número de vezes ao dia de refeição; de fácil digestão; com aspectos agradáveis; respeitar seus hábitos adquiridos ao longo de sua vida (Rodrigues \& Diogo, 2000).

Rodrigues e Diogo (2000) identificam como sendo alguns dos fatores que interferem na alimentação do idoso: alteração digestiva; hábitos culturais e religiosos; fatores psicológicos; problemas de saúde e fatores socioeconômicos.

A ingestão de proteínas pelos idosos deve ser adequada e suficiente para diminuir a perda muscular relacionada com a idade, porém deve-se ter em consideração que a ingestão elevada de proteínas pode facilitar os distúrbios renais, que são mais frequentes nessa faixa etária (Sousa et al., 2009).

Silva e Sá (2012), em estudo para identificar o nível de conhecimento em periódicos brasileiros e estrangeiros sobre Alimentos Funcionais em Idosos, concluíram que estes alimentos podem ajudar a minimizar algumas patologias comuns na terceira idade, e que podem levar a minimização de doenças como hepáticas, cardiovasculares, câncer entre outras, através dos compostos bioativos e substâncias que alguns alimentos contêm.

\section{Qualidade De Vida Da Pessoa Idosa}

Qualidade de vida é "a percepção que o indivíduo tem de sua posição na vida dentro do contexto de sua cultura e do sistema de valores de onde vive, e em relação a seus objetivos, expectativas, padrões e preocupações. É um conceito muito amplo que incorpora de uma maneira complexa a saúde física de uma pessoa, seu estado psicológico, seu nível de dependência, suas relações sociais, suas crenças e sua relação com características proeminentes no ambiente" (OMS, 1994).

A Organização Mundial de Saúde (1998) definiu qualidade de vida como a percepção do indivíduo de sua posição na vida, no contexto da cultura e do sistema de valores nos quais ele vive, em relação aos seus objetivos, expectativas, padrões e preocupações. No processo de envelhecimento, são analisados e avaliados os seis domínios propostos pelo grupo WHOQOL, a saber: físico, psicológico, nível de independência, relações sociais, meio-ambiente e espiritualidade (religião e crenças pessoais).

O conceito de qualidade de vida relaciona-se à autoestima e ao bem-estar pessoal e abrange uma grande gama de aspectos, tais como: capacidade funcional, nível socioeconômico, estado emocional, interação social, atividade intelectual, autocuidado, suporte familiar, estado de saúde, valores culturais, éticos e religiosidade, estilo de vida, satisfação com o emprego e/ou com as atividades da vida diária e com o ambiente em que se vive (Vecchia, 2005).

Assim, o conceito de qualidade de vida é subjetivo e dependente do nível sociocultural, da idade e das aspirações pessoais de cada indivíduo (Neri, 2007b, 2007c; Vecchia et al., 2005). 
Borglin et al. (2005) demonstraram que a qualidade de vida de idosos depende da preservação do self e da manutenção de objetivos ao longo da vida, e a experiência de qualidade de vida envolveria valores pessoais, experiências anteriores, capacidade de adaptação às mudanças, independência, autonomia, atividades, saúde, relações sociais e viver em casa.

Ruga (2003) investigou a alimentação de idosas, revelando hábitos alimentares inapropriados para a idade. A pesquisa constatou que $66,5 \%$ das entrevistadas não se alimentam adequadamente e apenas $6 \%$ consomem carne diariamente; tais percentuais são considerados extremamente baixos para um universo de pessoas de classe média e com bom nível de escolaridade. Segundo Ruga (2003), uma das explicações para o baixo índice de consumo de alimentos essenciais para a terceira idade seria o fator social: falta de motivação para preparar a alimentação e a desmotivação psicológica das idosas, causada por isolamento, depressão e perdas.

Envelhecer de forma ativa e ocupar-se com atividades prazerosas favorecem as pessoas longevas a uma vida com mais qualidade. A pessoa deve se envolver espontaneamente com atividades ocupacionais que favoreçam a sua participação social voluntária, bem como a sua capacidade criativa, sejam essas para repousar, divertir-se ou recrear-se (Mari et al., 2016).

Segundo Tramontino et al. (2009), o envelhecimento com qualidade de vida resulta do estilo de vida no qual cada pessoa opta em seguir, sendo os hábitos alimentares de grande influência sobre estes.

Oliveira et al (2010) sugerem que a prática de exercício físico pode ajudar as pessoas a manterem o maior vigor possível, melhorar a função em diversas atividades, aumentando a qualidade de vida à medida que envelhecem. Embora estas autoras salientem que as vantagens da prática de exercício físico pelas pessoas idosas dependem da forma como decorre o processo de envelhecimento e da rotina de exercício físico praticado, sabe-se que existem benefícios para a saúde mesmo quando a prática de exercício físico é iniciada numa fase tardia da vida.

Para Farenzena (2007), é impossível estudar o processo de envelhecimento sem abordar a qualidade de vida. A qualidade de vida é uma importante medida de impacto na saúde, por este motivo tem sido utilizada por profissionais de saúde, investigadores, economistas, administradores e políticos (Campos \& Neto, 2008).

\section{Considerações Finais}

A prática de exercício físico e hábitos alimentares saudáveis devem ser estimuladas nos Idosos, tendo em vista seu comprovado impacto na qualidade de vida destes. Ademais, através da análise da literatura, evidenciou-se os diversos fatores de riscos e problemas relacionados à ausência de uma boa alimentação e exercício físico, como maior incidência de doenças cardiovasculares, funções respiratórias reduzidas, sistema imune prejudicado, resultando em maior suscetibilidade à diversas outras doenças, principalmente na população Idosa.

Frente ao exposto, sugere-se a realização de novas pesquisas sobre a temática, especialmente pesquisas que possibilitem à pessoa idosa expor suas vivências através de relatos gravados para que os dados subjetivos possam servir como evidências científicas para reafirmar a importância dos cuidados em saúde para além do cuidado medicamentoso.

\section{Referências}

Alvino, F. S. (2015). Concepções do Idoso em um País que envelhece: Reflexões sobre protagonismo, cidadania e direitos humanos no envelhecimento, Dissertação (Mestrado em Direitos Humanos e Cidadania) — Universidade de Brasília, Brasília.

Amabis, J. M., et al. (2013). Os desafios da escola Pública Paranaense na Perspectiva do professor PDE; Caderno PDE, 1, 1-19.

Brito, F. C., \& Litvoc, J. (2004). Envelhecimento: prevenção e promoção de saúde. Atheneu.

Campos, M. O., \& Neto, R. F. J. (2008). Qualidade de vida: um instrumento para a promoção de saúde, Revista baiana de Saúde Pública, 32(2), 234-240.

Cancela, D. M. G. (2007). O processo de Envelhecimento, O portal dos psicólogos, 8. 
Carvalho, G. G. et al. (2008). Avaliação dos Hábitos Alimentares em idosos e Formulação de um Produto Alimentício. UNIVAP.

Caromano, F. M., et al. (2006). Manutenção na prática de exercícios por Idosos, Revista do Departamento de Psicologia-UFF, 18(2), 177-192.

Cunha, S. J., et al. (2019). Produção Científica Acerca Da Assistência De Enfermagem No Envelhecimento Saudável Da Pessoa Idosa: Revisão Integrativa, Faculdade de Enfermagem de São Vicente de Paula.

Doherty, T (2003). Invited review: Agingand sarcopenia. Journalof Applied Physiology, Washington, 95(4), $1717-27$.

Farenzena, P. W. (2007). Qualidade de vida em um grupo de idosos de Veranópolis-RS, Universidade católica do Rio grande do Sul, 1 -74.

Ferraz, et al. (2021). Impactos dos fatores extrínsecos no envelhecimento precoce: Uma reflexão teórica. Research, Society and Development, 10(6), e21210615761. http://dx.doi.org/10.33448/rsd-v10i6.15761

Flores, T. R., et al. (2018). Aconselhamento por profissionais de saúde e comportamentos saudáveis entre idosos: estudo de base populacional em Pelotas, Sul do Brasil.

Fontaine R (2000). Psicologia do envelhecimento. Climepsi Editores.

Gil, A. C. (2017). Como elaborar projetos de pesquisa. (6a ed.), Atlas.

Kalache, A., \& Jernigan, D. H. (2005). Envelhecimento ativo: Uma política de Saúde; Secretária de Vigilância em Saúde do Ministério da Saúde; Brazilia/DF, $7-57$.

Mari, et al (2016). O processo de envelhecimento e a saúde: o que pensam as pessoas de meia-idade sobre o tema, Rev. Bras. Geriatr. Gerontol.

Martinelli, S. S., \& Cavalli, B. S. (2019). Alimentação saudável e sustentável: uma revisão narrativa sobre desafios e perspectivas, Ciência Saúde coletiva.

Minayo, M. C. S. (2001). Pesquisa Social. Teoria, método e criatividade. (18a ed.), Petrópolis.

Moraes, E. M., et al. (2010). Características biológicas e psicológicas de envelhecimento. Rev Med Minas Gerais. 20(1). 67-73.

Organização Mundial de Saúde (OMS) (2015). Relatório mundial de envelhecimento e saúde. United States of America: Organização Mundial de Saúde. https://apps.who.int/iris/bitstream/handle/10665/186468/WHO_FWC_ALC_15.01_por.pdf\%3Bjse

OPAS. OPAS atualiza as funções essenciais de saúde pública para países das Americas,: OPAS atualiza funções essenciais de saúde pública para países das Américas - OPAS/OMS | Organização Pan-Americana da Saúde (paho.org).

Prado, et al. (2021). Exercício físico como ferramenta para o envelhecimento ativo: Uma reflexão teórica. Research, Society and Development, v. 10, n. 7 , e37710716629 http://dx.doi.org/10.33448/rsd-v10i7.16629

Ribeiro, B. A. J., et al. (2012). Adesão de Idosos a programas de atividade física: motivação e significância, Revista Brasileira de ciências e Esportes, 34, 969984.

Rodrigues, R. A. P., \& Diogo, M. J. D. E. (2000). Como cuidar dos idosos. Coleção Viva Idade.

Salgado, J. M. (2004). A alimentação que previne doenças: do pré-escolar à adolescência. Madras.

Santos, G. B., et al. (2016). Caracterização da saúde de idosos numa perspectiva fonoaudiológica. http://dx.doi.org/10.1590/1982-021620161855016;

Schneider, H. R. (2008). O envelhecimento na atualidade: Aspectos cronológicos, biológicos, Psicológicos e Sociais. Estudo de Psicologia.

Silva, M. R. et al. (2018). Estudos Qualitativos: Enfoques Teóricos e Técnicas de Coleta de Informações, UVA.

Sanavita (2003). Alimentação e Qualidade de Vida na Terceira Idade. Centro de Pesquisa Sanavita. Fundação de Gastroenterologia e Nutrição de São Paulo, São Paulo.

Sousa, A G., et al. (2011). Perfil sociodemográfico e nutricional de servidores em período de pré-aposentadoria. Rev. Pesq. Saúde, 12 , 16-21.

Silva, C. M. I., \& Sá, C. Q. E. (2012). Alimentos funcionais: um enfoque gerontológico, Rev Bras Clin Med, 10(1):24-8. 